\title{
The Ongoing Outbreak and Challenges of Novel Coronavirus (COVID-19) in China
}

\author{
Bohao Chen* \\ Section of Pulmonary and Critical Care Medicine, Department of Medicine, University of Chicago, Chicago, IL, USA
}

In December 2019, a cluster of unexplained cases of pneumonia was first reported by the Health Commission of Hubei Province, China. Most of cases were epidemiologically linked to a seafood and wet animal wholesale market in Wuhan, Hubei Province, China, which was officially closed on January $1,2020 .^{1}$ As of January 3 , the national authorities in China had reported a total of 44 patients with pneumonia to WHO. On January 8, virologists and other public health investigators identified a new coronavirus, now known as COVID-19, as the cause of the pneumonia clusters and posted the genetic sequence of COVID-19 on January $12 .{ }^{2}$ The clusters of new cases among families and the infection of 16 healthcare professionals indicate human-to-human transmission of the virus. ${ }^{3}$ Chinese public health authorities have quarantined travel from Wuhan to limit the spread of the virus since January 23, and since then, more Chinese cities have also been isolated. As new cases and death toll from COVID-19 in China escalate rapidly and with COVID-19 spreading to other countries, WHO Director-General Tedros Adhanom Ghebreyesus declared the COVID-19 outbreak a public health emergency of international concern on January 30, noting the potential spread of the virus to countries with weak health systems. The Trump administration announced on January 31 that it would temporarily bar foreigners from entering the US if they had been to China within the past 14 days. Compared to prior SARS-CoV in 2003 and MERS-CoV in 2013, the ongoing outbreak of COVID-19 presents an urgent and huge health threat since it is far more transmittable and far more damaging economically and socially. Therefore, governments and partners should take immediate actions in response to the COVID-19 outbreak and bring the scientific community together to accelerate research and innovation against the global epidemic of COVID-19.

Coronaviruses are single-stranded positive-sense RNA viruses and classified into four main sub-groupings, known as alpha, beta, gamma, and delta, based on antigenic relationships and viral genetic phylogeny. The former two genera primarily infect the respiratory and gastrointestinal tract of mammals, whereas the latter

\footnotetext{
Abbreviations: ACE2, angiotensin-converting enzyme 2; COVID-19, novel coronavirus; FDA, Food and Drug Administration; $\mathrm{HCoV}$, human coronavirus; MERS-CoV Middle East Respiratory Syndrome Coronavirus; RBD, receptor binding domain; SARS-CoV, Severe Acute Respiratory Syndrome Coronavirus; WHO, World Health Organization.

Received: February 17, 2020; Revised: February 21, 2020; Accepted: February 22, 2020

*Correspondence to: Bohao Chen, Section of Pulmonary and Critical Care Medicine, Department of Medicine, University of Chicago, 5841 S. Maryland Ave., Chicago, IL 60637, USA. Tel: 001-773-834-7476, Fax: 001-773-702-4736,

E-mail: bchen@medicine.bsd.uchicago.edu

How to cite this article: Chen B. The Ongoing Outbreak and Challenges of Nove Coronavirus (COVID-19) in China. Exploratory Research and Hypothesis in Medicine 2020;5(1):3-5. doi: 10.14218/ERHM.2020.00008.
}

two infect avian species. The genus Alphacoronavirus contains the human viruses HCoV-229E, HCoV-NL63, and many other animal viruses. The genus Betacoronavirus includes the human viruses HCoV-OC43 and HCoV-HKU1 along with a number of animal coronaviruses. Some coronaviruses that infect animals can evolve to acquire efficient human transmission, making people sick and becoming a new human coronavirus, such as SARS-CoV, MERS$\mathrm{CoV}$, and now COVID-19. ${ }^{4}$ In the 2003 SARS pandemic, investigators found SARS-CoV viral RNA in both palm civets and raccoon dogs in wet markets. It is very likely that SARS-CoV was transmitted from bats to those animals which served as intermediary reservoirs and then finally infected humans. ${ }^{5}$ MERS-CoV is also a zoonotic virus with possible origins in bats and camels. The ongoing outbreak of COVID-19 is associated with the Wuhan Huanan Seafood Market where snakes, birds, marmots, pangolin, and other small mammals were sold, suggesting animal-to-person spread. However, as the outbreak progressed, most cases were confirmed without exposure to animal markets, and cases were identified among healthcare professionals and other contacts of patients with COVID-19 infections, indicating either human to human transmission or a more widespread animal source. ${ }^{3}$ COVID-19 is highly contagious and the current epidemic has become a serious public health threat to China as well as to other countries. It requires governments to take emergency and mandatory measures to treat the patients, isolate the suspicious cases, protect healthcare professionals, track the spread of the virus, and advise individuals for public health.

So far, the source of COVID-19 has not been identified yet. Since the sequencing of COVID-19 genome by Dr. Zhang's group at Fudan University was published on January 12, 44 COVID-19 sequences have been deposited in the NCBI Virus website. Genome comparison of all these strains found that they are almost identical with $>99 \%$ sequence conservation. The low variability suggests a likely single emergence of epidemic viruses from a common animal reservoir. The COVID-19 genome (MN908947.3) shows similarities to Bat SARS-like coronavirus (CoVZC45) with sequence identity of $89.12 \%$ and to Bat SARS-like coronavirus (CoVZXC21) with sequence identity of $88.68 \%$. However, COVID-19 is genetically distinct from the human SARS genome with $82.3 \%$ sequence identity, supporting the claim that human COVID-19 is a zoonotic virus with possible origins from bats to human. ${ }^{6}$ However, no bats were sold at the Huanan seafood market, suggesting that another yet-to-be-identified animal acted as an intermediate to transmit the virus to humans. It is urgently requested that researchers clarify the source of $2019-\mathrm{nCoV}$ including possible intermediate animal vectors in order to end the COVID-19 spread.

Coronaviruses encode five structural proteins in their genomes. These are the spike surface glycoprotein (S), membrane protein $(\mathrm{M})$, nucleocapsid protein $(\mathrm{N})$, small envelope protein $(\mathrm{E})$, and 
Hemagglutinin Esterase protein (HE). All envelope proteins and $\mathrm{N}$ protein are present in all virions, but $\mathrm{HE}$ is only present in some beta coronaviruses. The spike surface glycoprotein plays an essential role in engaging with the host cell receptor ACE2 and mediating fusion between the viral and host cell membranes. The alignment analysis found S protein of COVID-19 shares $97.41 \%$ of amino acid identity with Bat coronavirus (QHR63300.1), 80.32\% with Bat SARS-like coronavirus (AVP78042.1), and 76.12\% with SARS coronavirus (PC4-137 AAV49720.1). The modeling and subsequent reports provide strong evidence that the $\mathrm{S}$ protein of COVID-19 has sufficient affinity with the SARS ACE2 receptor to infect bronchial epithelial cells and type II pneumocytes. ${ }^{7}$ However, there is much more to learn about the association of affinity of the ACE2 receptor with the transmissibility and pathogenesis of COVID-19, and more investigations will need to be done to block the virus invasion.

For SARS-CoV, the $\mathrm{S}$ protein is the main antigenic component that is responsible for inducing host immune responses, neutralizing antibodies and/or protective immunity against virus infection. $\mathrm{S}$ protein has therefore been selected as a major target for vaccine development and anti-viral therapy. Such efforts include using fulllength (Novavax, Phase III) or recombinant S protein (Vaxine Pty Ltd, Australia, Phase I) to induce effective neutralizing-antibodies and protective immunity, RBD-ACE2 blockers to block RBDACE2 binding and S protein-mediated infection, small interfering RNAs to reduce virus replication and/or silence $\mathrm{S}$ gene expression, inactivated or live attenuated vaccine, etc. $^{8}$ Although the feasibility of using the above approaches is partially limited by their low antiviral potency, studies and clinical trials on the SARS vaccine provide important information for designing novel strategies for prophylaxis and therapies against emerging infections caused by COVID $-19 .{ }^{7}$ We urge the government and private industry to grant additional funding to advance anti-coronavirus vaccines into clinical trials.

The symptoms and signs of COVID-19 infection are recently described in multiple clinical observations and include fever, cough, nasal congestion, fatigue, dyspnea, and significant changes visible through chest X-rays and computer tomography techniques (ground glass abnormalities, patchy consolidation, alveolar exudates, and interlobular involvement). Cases with gastrointestinal symptoms and asymptomatic infections have also been reported recently, especially among young children. ${ }^{9}$ Statistical analysis showed that the highest incidence of COVID-19 was observed among adults older than 50 years, with the lowest incidence in the age group younger than 20 years. Furthermore, males experienced a higher incidence than females. The deaths also were concentrated in male patients over 60 years of age with severe pneumonia at diagnosis. ${ }^{10}$ It is unclear yet why some infections have no symptoms while others show severe pneumonia and even death. More research should be conducted on the interactions among viral virulence determinants, the density and the affinity of ACE2 receptor on cell surface, and the host's immune response.

Clinical treatment of infections with COVID-19 is still based on supportive care, like oxygen therapy and fluid management, which has proven to be very effective. Encouraging preliminary trials indicate that infusions of blood plasma from people who have recovered from COVID-19 could help the critically ill patients improve clinical symptoms. Although antiviral drugs have made great progress in recent years, currently there are no therapeutic agents licensed and available for COVID-19. In theory, some anti-SARS-CoV and anti-Ebola virus drugs in clinical trials should have activity against COVID-19. In the current outbreak of COVID-19, the WHO prioritized a panel of candidate thera- peutics, including antibodies, nucleoside analogues, and protease inhibitors for repurposing under FDA Emergency Use Authorization. Chinese experts are considering a flexible design to offer investigational therapeutics to those infected with COVID-19 and evaluate the safety and efficacy of these drugs on an emergency basis. The government and the private sectors should provide more financial assistance for the scientific community to design or develop novel and specific antiviral countermeasures for emerging coronaviruses.

The COVID-19 epidemic is still going on in China and is far from over. As of February 15, China had reported more than 68,594 confirmed cases, 1,716 of which are medical professionals. The death toll from COVID-19 had risen to 1,667 with 11,272 critically ill patients. Under the transportation shutdown of Wuhan City and severe shortages of medical resources, Chinese authorities face daunting challenges to provide appropriate medical treatment for more than 68,000 patients, protect thousands of healthcare professionals from nosocomial infection, quarantine millions of people, and deal with the socioeconomic impacts of the epidemic. This problem requires international engagement and cooperation against possible COVID-19 global pandemic.

\section{Acknowledgments}

The author thanks all investigators and clinicians who have contributed to our understanding of COVID-19 infections.

\section{Funding}

None to declare.

\section{Conflict of interest}

The author has no conflict of interests related to this publication.

\section{References}

[1] Huang C, Wang Y, Li X, Ren L, Zhao J, Hu Y, et al. Clinical features of patients infected with 2019 novel coronavirus in Wuhan, China. Lancet 2020;395(10223):497-506. doi:10.1016/S0140-6736(20)30183-5.

[2] Zhu N, Zhang D, Wang W, Li X, Yang B, Song J, et al. A Novel Coronavirus from Patients with Pneumonia in China, 2019. N Engl J Med 2020;382(8):727-733. doi:10.1056/NEJMoa2001017.

[3] Phan LT, Nguyen TV, Luong QC, Nguyen TV, Nguyen HT, Le HQ, et al. Importation and Human-to-Human Transmission of a Novel Coronavirus in Vietnam. N Engl J Med 2020;382(9):872-874. doi:10.1056/ NEJMc2001272.

[4] Wang Q, Qi J, Yuan $Y, X$ Xuan $Y$, Han P, Wan Y, et al. Bat origins of MERS-CoV supported by bat coronavirus HKU4 usage of human receptor CD26. Cell Host Microbe 2014;16(3):328-337. doi:10.1016/j. chom.2014.08.009.

[5] Kan B, Wang M, Jing H, Xu H, Jiang X, Yan M, et al. Molecular evolution analysis and geographic investigation of severe acute respiratory syndrome coronavirus-like virus in palm civets at an animal market and on farms. J Virol 2005;79(18):11892-11900. doi:10.1128/ JVI.79.18.11892-11900.2005.

[6] Gralinski LE, Menachery VD. Return of the Coronavirus: 2019-nCoV. Viruses 2020;12(2):E135. doi:10.3390/v12020135.

[7] Wan Y, Shang J, Graham R, Baric RS, Li F. Receptor recognition by novel coronavirus from Wuhan: An analysis based on decade-long structural studies of SARS. J Virol 2020;JVI.00127-20. Epub 2020 Jan 
29. doi:10.1128/JVI.00127-20.

[8] Du L, He Y, Zhou Y, Liu S, Zheng BJ, Jiang S. The spike protein of SARS$\mathrm{CoV}-\mathrm{a}$ target for vaccine and therapeutic development. Nat Rev Microbiol 2009;7(3):226-236. doi:10.1038/nrmicro2090.

[9] Shi H, Han X, Zheng C. Evolution of CT Manifestations in a Patient Recovered from 2019 Novel Coronavirus (2019-nCoV) Pneumonia in
Wuhan, China. Radiology 2020;200269. Epub Feb 7. doi:10.1148/radiol.2020200269.

[10] Chen N, Zhou M, Dong X, Qu J, Gong F, Han Y, et al. Epidemiological and clinical characteristics of 99 cases of 2019 novel coronavirus pneumonia in Wuhan, China: a descriptive study. Lancet 2020;395(10223):507-513. doi:10.1016/S0140-6736(20)30211-7. 